\title{
Xenogeneic chondrocytes promote stable subcutaneous chondrogenesis of bone marrow-derived stromal cells
}

\author{
KE XUE $^{1}$, YUEQIAN ZHU ${ }^{1}$, YINGYING ZHANG ${ }^{1}$, CHENGAN CHIANG $^{1}$, \\ GUANGDONG ZHOU ${ }^{1-3}$ and KAI LIU ${ }^{1}$

\begin{abstract}
${ }^{1}$ Department of Plastic and Reconstructive Surgery, Shanghai Ninth People's Hospital, Shanghai Jiao Tong University School of Medicine, Shanghai Key Laboratory of Tissue Engineering, Shanghai 200011; ${ }^{2}$ Shanghai Stem Cell Institute, Shanghai 200020; ${ }^{3}$ National Tissue Engineering Center of China, Shanghai 200025, P.R. China
\end{abstract}

Received September 6, 2011; Accepted October 20, 2011

DOI: $10.3892 /$ ijmm.2011.830

\begin{abstract}
The local microenvironment may change the ultimate fate of engineered cartilage differentiated from bone marrow stromal cells (BMSCs) after subcutaneous implantation. Chondrogenically differentiated BMSCs directed by growth factors or low-intensity ultrasound are apt to fibrose or vascularize in the subcutaneous environment, while BMSCs implanted in articular cartilage defects can form stable cartilage. We hypothesized that chondrocytes would provide an ideal chondrogenic environment, and thus promote the maintenance of the chondrocytic phenotype in ectopia. To test this hypothesis, we developed a new method to promote chondrocyte development from BMSCs in a chondrogenic environment produced by xenogeneic chondrocytes and compared the subcutaneous chondrogenesis of BMSCs mediated by xenogeneic chondrocytes with that produced by growth factors. These results indicate that subcutaneous chondrogenesis of BMSCs directed by xenogeneic chondrocytes is more effective than that induced by growth factors. BMSCs induced by xenogeneic chondrocytes formed relatively mature cartilage before or after implantation, following 4 weeks of culture, which reduced the induction time in vitro and led to maintenance of a stable cartilage phenotype after subcutaneous implantation.
\end{abstract}

\section{Introduction}

Bone marrow stromal cells (BMSCs) are an attractive source of seeding cells for tissue engineering due to their obvious

Correspondence to: Dr Kai Liu, Department of Plastic and Reconstructive Surgery, Shanghai Ninth People's Hospital, Shanghai Jiao Tong University School of Medicine, Shanghai Key Laboratory of Tissue Engineering, 639 Zhizaoju Road, Shanghai 200011, P.R. China

E-mail: drkailiu@126.com

Key words: xenogeneic chondrocytes, bone marrow stromal cells, co-culture, subcutaneous chondrogenesis advantages: autologous acquisition, multilineage differentiation potential and high proliferation potential in vitro. BMSCs can differentiate into osteogenic, chondrogenic, and adipogenic lineages (1), and they can be combined with scaffold material to allow repair of articular cartilage defects, and differentiate into chondrocytes or osteoblasts in different joint microenvironments $(2,3)$.

However, a significant amount of time and money is required for induction of chondrocyte differentiation from BMSCs in vitro using growth factors. In addition, engineered cartilage differentiated from BMSCs does not maintain a stable chondrogenic phenotype after subcutaneous implantation, although it does exhibit a chondrocyte phenotype beforehand (4-6). Engineered cartilage has been found to ossify after implantation while chondrogenic induction lasts 8 weeks or less in vitro (7). These results may limit the feasibility of using MSCs to repair cartilage defects in the subcutaneous environment since most cartilage injuries occur in a subcutaneous area, such as trauma of the nasal or auricle cartilage. However, BMSCs are able to form stable cartilage within defects in articular cartilage, and they have also been shown to form stable cartilage-like tissue in a subcutaneous environment when co-cultured with autogeneic chondrocytes (8). Therefore, we speculated that the microenvironment produced by chondrocytes plays an important role in determining the ultimate fate of engineered cartilage differentiated from BMSCs after subcutaneous implantation, and that the presence of autogeneic chondrocytes regulates the chondrocytic differentiation of BMSCs. However, autogeneic co-culture (direct mixing of autogeneic chondrocytes and BMSCs to construct cartilage) carries the cost of sacrificing autogeneic chondrocytes which are rare and hard to obtain, so they cannot provide sufficient material to adequately repair autologous cartilage defects. In contrast, a rich source of xenogeneic chondrocytes is readily available, hence, we hypothesized that using xenogeneic chondrocytes instead of autogeneic chondrocytes would promote the chondrogenesis of BMSCs, which would then retain a stable chondrocytic phenotype after subcutaneous implantation. To test this hypothesis, we designed an isolated xenogeneic co-culture system and compared subcutaneous chondrogenesis of BMSCs induced by xenogeneic chondrocytes with that of BMSCs induced by growth factors. 


\section{Materials and methods}

All animal experimental procedures in this study were approved by the Ethics Committee of the Shanghai Jiao Tong University School of Medicine. Swine bone marrow was obtained from the posterior superior iliac crests of newborn pigs. Rabbit cartilage specimens were obtained from the ear cartilage of 2-month-old New Zealand White rabbits.

Cell harvest and culture. BMSCs from newborn pigs and articular chondrocytes from New Zealand White rabbits were isolated and expanded as previously described $(9,10)$. Swine BMSCs at passage 2 were used for tissue engineering, and rabbit chondrocytes at passage 2 were used in culture, a portion of the chondrocytes at passage 1 were cryopreserved for later use.

Preparation of scaffold constructs and cell seeding. Polylactic acid-(PLA-) coated polyglycolic acid-(PGA) scaffolds were prepared as described previously (10). Briefly, $7 \mathrm{mg}$ of PGA unwoven fibers (Dong Hua University, China) were compressed into a disc of $5 \mathrm{~mm}$ diameter and $2 \mathrm{~mm}$ thickness, and $0.15 \mathrm{ml}$ of $1.0 \%$ PLA (Sigma) diluted in dichloromethane was used to solidify each scaffold. The scaffolds were sterilized by soaking in 75\% alcohol for $1 \mathrm{~h}$ and washed 3 times with PBS, then precultured overnight in Dulbecco's modified Eagle's medium (DMEM) in an incubator at $37^{\circ} \mathrm{C}$ in an atmosphere of $5 \% \mathrm{CO}_{2}$, 95\% air, and $99 \%$ relative humidity.

A $30 \mu 1$ volume of a suspension of swine BMSCs at passage $2\left(5.0 \times 10^{7} / \mathrm{ml}\right)$ was evenly applied dropwise onto each scaffold. After incubation for 4-6 $\mathrm{h}$ to allow preliminary adhesion of the cells to the scaffold, $3 \mathrm{ml}$ of regular culture medium was added, and the constructs were then returned to the incubator. After incubating for $24 \mathrm{~h}$ to allow complete adhesion, sufficient regular culture medium was added to cover the cell-scaffold construct.

Chondrogenic induction in vitro. After 5 days of culture, BMSC-scaffold constructs were divided into 3 groups: xenogeneic chondrocyte group (EC group), growth factor group (GF group) and blank control group (BC group). In the $\mathrm{EC}$ group, the constructs were cultured in high glucose DMEM in a $0.4 \mu \mathrm{m}$ transwell chamber, with adherent rabbit chondrocytes cultured in the dish below the membrane. Half of the culture medium was changed every other day, and the dish was replaced with a new culture dish containing new adherent rabbit chondrocytes (obtained from recovery of previously cryopreserved chondrocytes) every week. In the GF group, the culture medium was replaced with chondrogenic induction medium composed of regular culture medium supplemented with $10 \mathrm{ng} / \mathrm{ml}$ transforming growth factor $\beta 1$ (TGF- $\beta 1$, Peprotech, Rocky Hill, NJ), $50 \mathrm{ng} / \mathrm{ml}$ insulin-like growth factor 1 (IGF-1, Peprotech) and $40 \mathrm{ng} / \mathrm{ml}$ dexamethasone (Sigma, St. Louis, MO) (11). Two-thirds of the culture medium was refreshed every other day. The attachment, proliferation and matrix production of the cells on scaffolds were examined using a light microscopy (Eclipse TS100, Nikon, Japan) and scanning electron microscopy (SEM, Philips XL-30, Amsterdam, The Netherlands). In the BC group, BMSC-PGA constructs were cultured in high glucose DMEM without chondrocytes or growth factors.
Subcutaneous implantation of engineered cartilage. After 2, 4, 6 and 8 weeks of in vitro chondrogenic induction, the constructs were implanted subcutaneously into nude mice and harvested at 6 weeks post-implantation.

Gross view, wet weight and GAG content of engineered tissue. Six weeks after implantation, the wet weight of the harvested specimens was measured using an electronic balance. The glycosaminoglycan (GAG) content of the specimens was assayed by Alcian Blue colorimetric analysis as previously described $(10,12)$.

Histology and immunohistochemistry. In order to evaluate the chondrogenesis of BMSCs in subcutaneous environments, the engineered tissues harvested after implantation were sent for histological and immunohistochemical examination. Hematoxylin and eosin (H\&E) and Safranin O staining, and type II collagen immunostaining, were used to evaluate the histological structure and cartilage matrix deposition in engineered tissue. Expression of type II collagen was detected by a mouse anti-human type II collagen monoclonal antibody (1:100 in PBS, Santa Cruz Biotechnology, Santa Cruz, CA, USA) followed by a horseradish peroxidase (HRP)conjugated anti-mouse secondary antibody (1:200 in PBS, Santa Cruz Biotechnology). Color development was performed with diaminobenzidine tetrahydrochloride (DAB, Santa Cruz Biotechnology).

Detection and comparison of TGF- $\beta 1$ in the supernatant of fresh or frozen monolayer chondrocyte cultures. It is known that TGF- $\beta 1$ plays an important role in chondrogenic differentiation of BMSCs, so we used ELISA to quantitatively analyze the amounts of TGF- $\beta 1$ in the supernatant of fresh or frozen chondrocytes at different times, then compared the amount of growth factors in the EC group with that in the GF group, and also assessed whether the growth factor secretion of chondrocytes differed after in vitro passage and anabiosis from the frozen store.

Statistical analysis. The difference in wet weight and GAG content of in vivo engineered tissues between the EC and the GF groups at each time point, and the differences within each group among various time points, were analyzed by the Student's t-test. Differences in TGF- $\beta 1$ levels in the supernatant of fresh and frozen chondrocytes at different times were also analyzed by a Student's t-test; a P-value $<0.05$ was considered statistically significant.

\section{Results}

Cell-scaffold construct culture in vitro. BMSCs at passage 2 were added dropwise to the scaffold for cartilage engineering. As described, a PGA scaffold with a relatively precise disc shape ( $5 \mathrm{~mm}$ in diameter, $2 \mathrm{~mm}$ in thickness) was formed. PLA was evenly coated on the surface of PGA fibers, which solidified the PGA fiber mesh and thus maintained the shape and porosity of the scaffold (Fig. 1A). After 7 days of culture, cells adhered well to the surface of the material, and produced abundant extracellular matrix in both the EC (Fig. 1B and C) and the GF groups (Fig. 1D). 

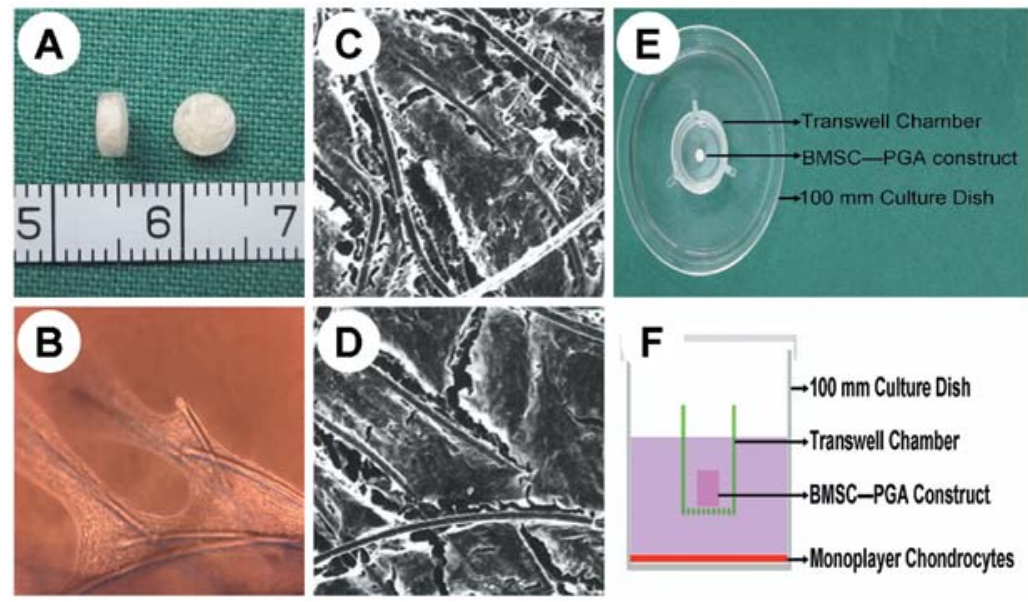

Figure 1. BMSC-scaffold constructs and the co-culture system. (A) PLA-coated PGA scaffold of $2 \mathrm{~mm}$ thickness and $5 \mathrm{~mm}$ diameter. (B) Light microscopy view of the scaffold, extracellular matrix can be observed in the EC group at the 7th day of culture. (C and D) SEM view of cell-scaffold constructs in the EC group and the GF group, showing good compatibility of the cells with the scaffold and abundant extracellular matrix production after 7 days of culture. Scale bar, $100 \mu \mathrm{m}$. (E and F) Schematic diagram of the isolated co-culture system. In this system, BMSC-PGA constructs are isolated from chondrocytes in a transwell chamber. Soluble factors secreted by each cell type can freely pass through the $0.4 \mu \mathrm{m}$ microporous membrane, while the cells cannot.

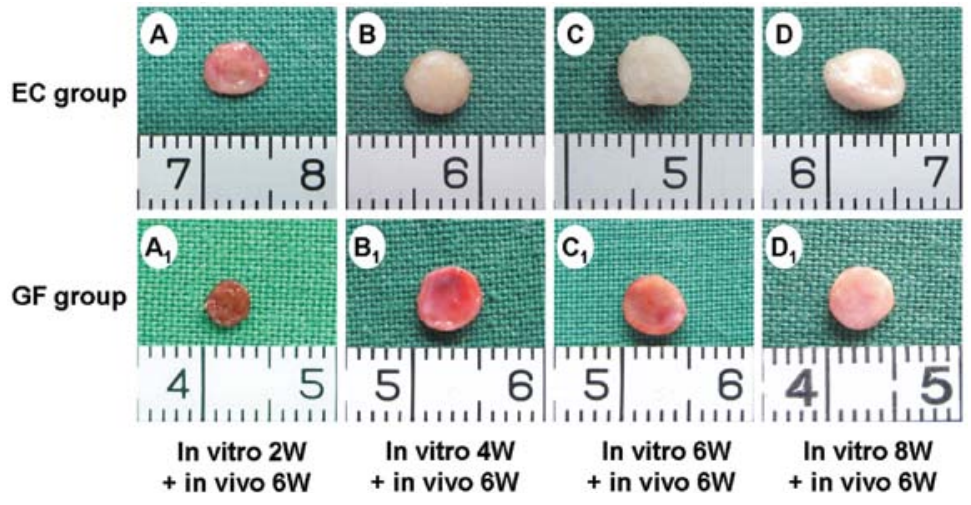

Figure 2. Gross view of engineered tissues after subcutaneous implantation in the EC and the GF groups. Most BMSC-scaffold constructs were able to form cartilage-like tissue, and only tissues which had undergone culture for 2 weeks followed by subcutaneous implantation for 6 weeks seemed to be a little vascularized (A-D) in the EC group. The appearance of engineered tissues in the GF group represented gradual development from a vascularized construct at 2 weeks to a light red and ivory-whitish cartilage-like tissue at 8 weeks $\left(A_{1}-D_{1}\right)$.

Chondrogenesis of BMSCs in vitro. In our previous and current study, we proved that a cell-scaffold construct grown in the presence of growth factors formed cartilage-like tissue after several weeks of induction in vitro, while in the BC group, only fibrous tissues were observed with no formation of a lacuna structure even after 8 weeks of culture. In the present study, we also performed histological and immunohistochemical examination before implantation in the EC group, and the results showed that a lacuna structure had formed in the specimens after culture for 2 (Fig. 3A-C) and 4 weeks (Fig. 3D-F).

Gross view, histology and immunohistochemistry. Six weeks after subcutaneous implantation, all the specimens in the EC group had formed cartilage-like tissue, while parts of the engineered tissues in the GF group had become obviously vascularized and fibrosed. In the EC group, only the cell-scaffold construct which had been cultured for 2 weeks prior to implantation seemed to be a little vascularized (Fig. 2A) while the rest showed an ivory-whitish appearance (Fig. 2B-D). However, the appearance of the GF group devel- oped gradually from a vascularized construct at 2 weeks to a cartilage-like tissue at 8 weeks after subcutaneous implantation (Fig. 2A $\mathrm{A}_{1}-\mathrm{D}_{1}$ ).

Histological findings revealed that the cell-scaffold construct in the EC group formed a heterogeneous tissue, containing cartilage-like tissue and fibrous tissue, and obvious lacuna-like structures were observed in the sample which had undergone 2 weeks culture in vitro followed by 6 weeks in vivo (Fig. 4A), although the new tissues were loose and incompact. These findings were further supported by Safranin O staining and type II collagen immunostaining (Fig. 4B and C). With increasing induction time in vitro, the majority of the cells formed lacunalike structures with positive staining for Safranin O and type II collagen. The constructs formed typical lacuna structures, and a more compact structure as well as stronger extracellular matrix staining were observed in those samples which underwent 4 weeks in vitro and 6 weeks in vivo, which was seen in samples of the GF group only after 8 weeks in vitro and 6 weeks in vivo (Fig. $4 \mathrm{~J}_{1}, \mathrm{~K}_{1}$ and $\mathrm{L}_{1}$ ). As induction time increased, more mature cartilage was formed. 


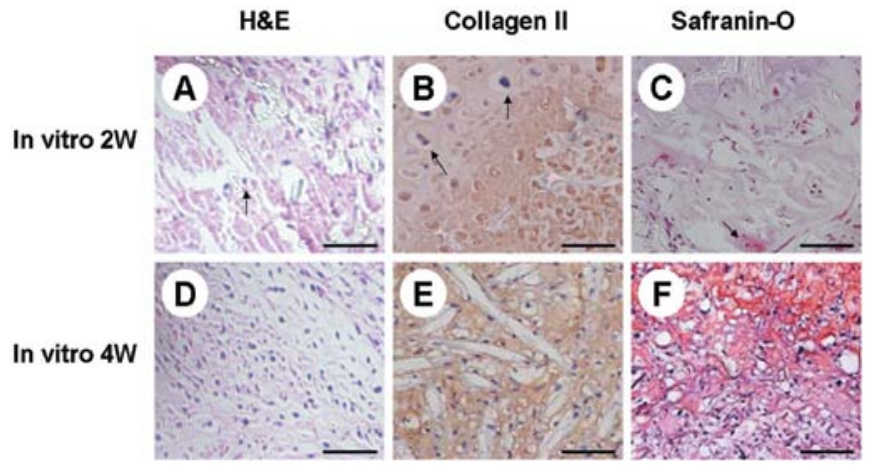

Figure 3. Histology, immunohistochemical and histochemical staining of in vitro engineered tissues in the EC group. After 2 weeks of induction, the construct formed cartilage-like tissue, and lacuna structures appeared (A-C, short arrow). After 4 weeks in vitro, relatively mature cartilage was formed, and many lacuna structures appeared. Scale bar, $100 \mu \mathrm{m}$.
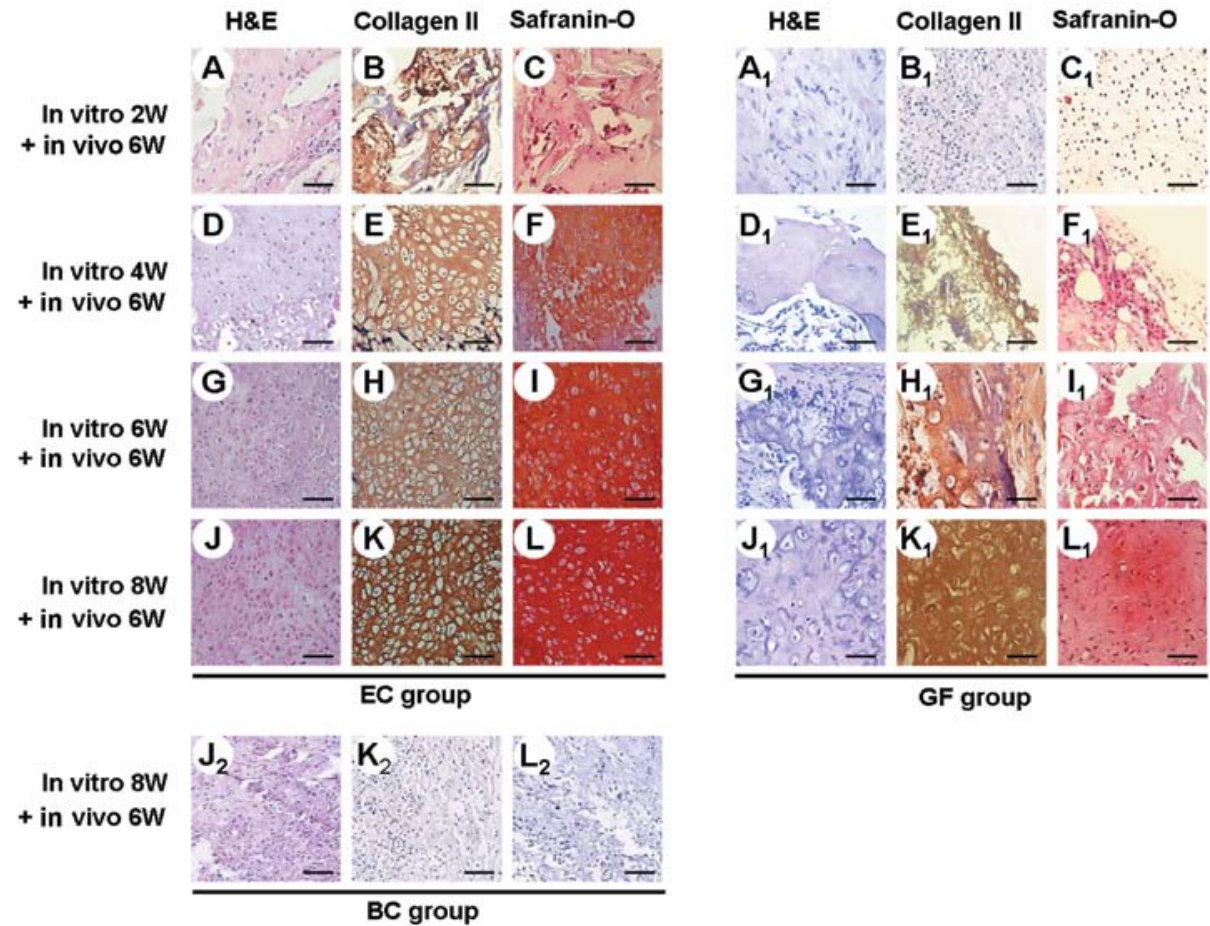

Figure 4. Comparison of chondrogenesis of engineered tissues after subcutaneous implantation. In the EC group, after subcutaneous implantation for 6 weeks, lacuna structures appeared (A) and positive staining with a type II collagen antibody (B) and Safranin O (C) were observed. The tissue engineered by 4-week induction in vitro mostly formed cartilage-like tissue with positive staining of type II collagen (E) and Safranin O (F) after implantation. With prolonged induction time, positive staining of Safranin O and type II collagen were enhanced. In the GF group, tissue engineered by 2 weeks of induction in vitro mainly underwent fibrosis after implantation, and immunohistochemical and histochemical staining showed no existence of cartilage-like tissue $\left(A_{1}-C_{1}\right)$. $A$ few sporadic lacuna-like structures appeared after 4 weeks of induction $\left(D_{1}-F_{1}\right)$. With extended induction time, the histological structure of the constructs gradually became more compact, more lacuna structures appeared and a trend of enhanced positive staining of type II collagen and Safranin $\mathrm{O}$ was observed. As expected, no lacuna structures were observed after implantation, even after 8 weeks of culture, only fibrous tissues were formed in the $\mathrm{BC}$ group $\left(\mathrm{J}_{2}, \mathrm{~K}_{2}\right.$ and $\mathrm{L}_{2}$ ). Scale bar, $100 \mu \mathrm{m}$.

In contrast, specimens in the GF group did not appear to form cartilage-like tissue as the specific staining was negative and no lacuna-like structures were found after 2 weeks in vitro and 6 weeks in vivo. A few occasional lacuna-like structures appeared up to 4 weeks in culture while more sporadic lacunalike structures formed with increasing culture time.

In the $\mathrm{BC}$ group, only fibrous tissues were observed with no formation of lacuna structures even after 8 weeks of culture, (Fig. $4 \mathrm{~J}_{2}, \mathrm{~K}_{2}$ and $\mathrm{L}_{2}$ ), indicating that no spontaneous chondrogenesis occurred in the absence of chondrogenic induction in the subcutaneous environment.
Wet weight and GAG content. The wet weight and GAG content $(\mathrm{n}=9)$ increased between 2 and 8 weeks in both the $\mathrm{EC}$ and the GF groups after implantation (Fig. 5). A significant difference in both wet weight and GAG content was found between these 2 groups at each time point $(\mathrm{P}<0.01)$, and in the GF group among the various time points. No significant increase of wet weight or GAG content was observed from 6 to 8 weeks in the EC group after implantation. The GAG content of the implanted constructs at 8 weeks in the EC group reached $94.9 \%$ of the normal auricular cartilage level, while that in the GF group reached $41.1 \%$ of the normal auricular cartilage level (Fig. 5B). 
A

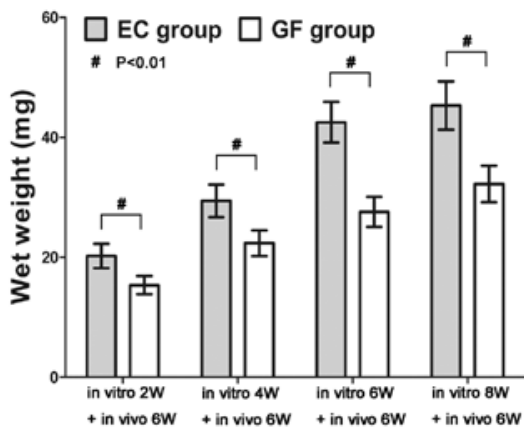

C

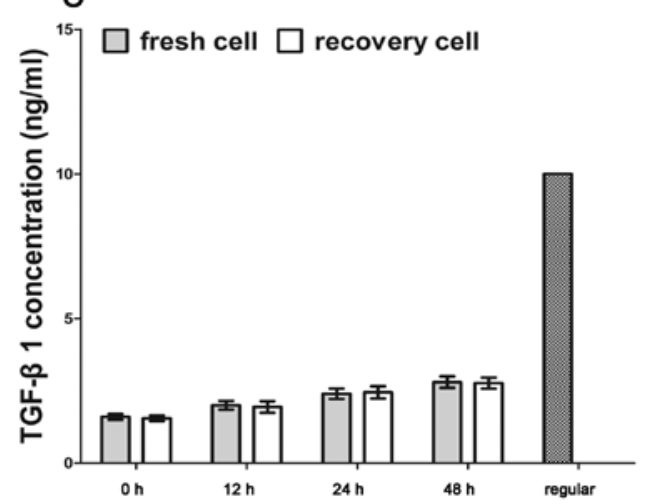

B

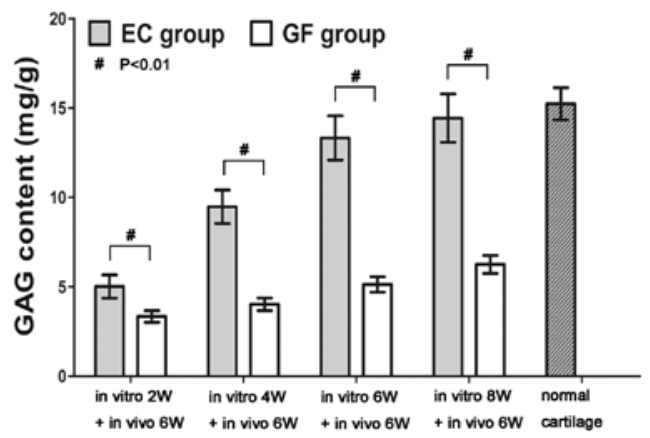

Figure 5. Wet weight and GAG content of engineered tissues following in vivo implantation, and the concentration of TGF- $\beta 1$ in the supernatant of fresh and recovered frozen chondrocytes. (A) Wet weight increased between 2 and 8 weeks in both the EC group and the GF group, and a significant difference in wet weight was found between these 2 groups at each time point $(\mathrm{P}<0.01)$ as well as among various time points in the GF group $(\mathrm{P}<0.05)$; however, wet weight in the EC group did not show any obvious difference after implantation between specimens induced in vitro for 6 or 8 weeks. (B) The GAG content in the 2 groups also increased gradually from 2 to 8 weeks with a significant difference among the various time points $(\mathrm{P}<0.01)$ and $\mathrm{GAG}$ content in the EC group was obviously higher than that in the GF group at each time point $(\mathrm{P}<0.01)$. The GAG content in the EC group showed the same trend as wet weight, with no significant difference among specimens after implantation following in vitro induction for 6 or 8 weeks, and the GAG content of implanted cartilage after 8 weeks in vitro induction reached $94.9 \%$ of the normal cartilage level. (C) The concentration of TGF- $\beta 1$ increased over time, and there was no obvious difference in the concentration in supernatants from fresh cells or from frozen chondrocytes after recovery. The concentrations secreted by chondrocytes were lower than the $10 \mathrm{ng} / \mathrm{ml}$ concentration observed in the regular chondrogenic induction medium used in the GF group cultures.

The important role of TGF- $\beta 1$ in chondrogenic differentiation of BMSCs. It is well known that growth factors play a very important role in chondrogenic differentiation of stem cells, and TGF- $\beta 1$ is the most widely used factor. In this study, we measured the amount of this growth factor in the supernatant of monolayer chondrocytes, and found that the concentrations increased over time. Although the concentrations of TGF- $\beta 1$ secreted by chondrocytes were lower than those used in the GF group, they still provided an optimal environment for chondrocytic differentiation of BMSCs. We therefore speculated that other factors apart from TGF- $\beta 1$ may be important in this process, since chondrocytic differentiation of BMSCs directed by chondrocytes appeared to be better than that induced by growth factors directly. In addition, in order to obtain the type of chondrogenic microenvironments provided by chondrocytes at any time, we hypothesized that frozen chondrocytes may also secrete the same amount of growth factors after anabiosis from the frozen store. Our results showed no obvious change in the concentration of TGF- $\beta 1$ in the supernatant of frozen chondrocytes after recovery (Fig. 5C).

\section{Discussion}

The theory that BMSCs can form cartilage-like tissue has been proven in a number of studies. In 1976, Freidenstein initially reported that in vitro, BMSCs could differentiate into osteogenic, chondrogenic, adipogenic and other lineages (13). Additionally, BMSCs were able to maintain their multilineage differentiation capacity after several passages. In the past 30 years, the pluripotency of BMSCs has been corroborated further by a series of experiments. In vitro, TGF- $\beta$ (including TGF- $\beta 1$, TGF- $\beta 2$ and TGF- $\beta 3$ ) is most often used in the induction of chondrogenesis from BMSCs $(11,14)$, although other growth factors or reagents such as IGF, BMP-2, or dexamethasone also seem to influence the promotion of chondrocytic differentiation of BMSCs. However, many problems still exist. For one thing, growth factors are expensive and in great demand. In addition, induction of cartilage in vitro takes a long time, and engineered cartilages are apt to become fibrosed, vascularized or ossified, and these constructs also eventually shrink to a certain degree $(4,6,7)$. Hence, the above-mentioned problems limit the clinical application of engineered cartilage.

Recently, however, we have successfully constructed cartilage using a co-culture system in which chondrocytes and BMSCs were mixed in a definite ratio to construct cartilage. More importantly, the engineered cartilage retained a stable cartilage phenotype after subcutaneous implantation $(8,15)$, offering a new method for cartilage engineering. However, autologous co-culture (mixing of autologous chondrocytes and 
BMSCs to construct cartilage) comes at the cost of sacrificing autologous chondrocytes, which violates the basic principles of 'non-invasive repair and minimally invasive repair' in tissue engineering. Although mature cartilage can be created by co-culture, its clinical application could be of no significance.

Bearing in mind all these issues, we designed an isolated xenogeneic co-culture system in our current study. We utilized xenogeneic chondrocytes instead of autologous chondrocytes in order to avoid self injury since xenogeneic chondrocytes are abundant and widely available. To further facilitate the acquisition of xenogeneic chondrocytes, we employed cell cryopreservation (16) and compared the amount of growth factors secreted by fresh and frozen chondrocytes, and our results indicate that frozen cells after recovery can provide an induction microenvironment as good as fresh cells. In addition, monolayer expanded chondrocytes lost their native morphology within 1 week (17), so to avoid loss of the chondrogenic microenvironment we chose to change the Petri dish, providing one containing new adherent chondrocytes every week, which guaranteed that the new cells secreted sufficient growth factors and other unknown factors. In order to solve immunogenicity problems, a transwell chamber with a microporous membrane of only $0.4 \mu \mathrm{m}$ was used, which allowed only the soluble factors to penetrate freely while keeping the BMSCs separate from the xenogeneic chondrocytes. Using this method, immunological rejection caused by xenogeneic chondrocytes can be greatly reduced.

Our results show that, compared with engineered tissues induced by growth factors, the cell-scaffold induced by xenogeneic chondrocytes tended to form cartilage tissue at an early stage of 2 weeks in vitro. After 4 weeks of in vitro induction, samples formed relatively mature cartilage, as observed by histological staining. Furthermore, engineered cartilage was able to retain a stable phenotype in the subcutaneous environment. Although the quantitative determination of the key growth factor TGF- $\beta 1$ known to be chondrogenic in the supernatant of chondrocytes demonstrated a lower concentration than that in the GF group, the improved chondrogenesis observed implies that some other unknown factors secreted by chondrocytes might also play a relatively important role in this process. All in all, in vitro induction time was reduced and relatively mature cartilage could be formed by this method.

Of course, the immunogenicity of foreign proteins needed to be taken into account, because growth factors secreted by xenogeneic cells can be considered foreign proteins and thus may cause immunologic reaction after autologous implantation for clinical application. With regard to this problem, some studies have shown that metabolism and degradation of foreign proteins would occur during further culture in a medium containing autologous proteins $(18,19)$, and foreign proteins would then be replaced by autologous proteins.

It has been proven that BMSCs possess a low immunogenic profile and potential and have immunomodulatory properties in both differentiated and undifferentiated states (20-22), which would reduce the risk of graft failure of chondrogenically differentiated BMSCs. In addition, according to the rules and regulations of the FDA in the USA, BMSCs cultured in FBS are widely permitted for use in clinical trials, and xenogeneic acellular dermal matrix used in the clinic (23-25). On the other hand, cartilage was eventually implanted into the subcutaneous environment, in which the immune response is relatively low, and in our next study, we are improving the method further to lower the immunogenicity and to test the engineered tissue in immune animals.

In summary, we confirmed that xenogeneic chondrocytes promoted the formation of relatively mature cartilage by BMSCs in a short time. More importantly, chondrogenically differentiated BMSCs were able to retain their stable cartilage structure after subcutaneous implantation, which was obviously better than the cartilage induced by growth factors. Although the exact mechanism remains unclear, cartilage differentiated in vitro from BMSCs by this new method could provide an alternative graft source for the functional repair of cartilage defects in the subcutaneous environment. In addition, the new method of cartilage engineering established in this study may be practical for a wider application in cartilage engineering and to promote its clinical application.

\section{Acknowledgements}

This study was supported by the National Natural Science Foundation of China (30872697). The authors appreciate the technical supports from Demin Yin, Lijuan Zong and Juanjuan $\mathrm{Wu}$ in the laboratory.

\section{References}

1. Pittenger MF, Mackay AM, Beck SC, Jaiswal RK, Douglas R, Mosca JD, Moorman MA, Simonetti DW, Craig S and Marshak DR: Multilineage potential of adult human mesenchymal stem cells. Science 284: 143-147, 1999.

2. Wakitani S, Goto T, Pineda SJ, Young RG, Mansour JM, Caplan AI and Goldberg VM: Mesenchymal cell-based repair of large, full-thickness defects of articular cartilage. J Bone Joint Surg Am 76: 579-592, 1994.

3. Grande DA, Southerland SS, Manji R, Pate DW, Schwartz RE and Lucas PA: Repair of articular cartilage defects using mesenchymal stem cells. Tissue Eng 1: 345-353, 1995.

4. De Bari C, Dell'Accio F and Luyten FP: Failure of in vitro-differentiated mesenchymal stem cells from the synovial membrane to form ectopic stable cartilage in vivo. Arthritis Rheum 50: 142-150, 2004.

5. Pelttari K, Winter A, Steck E, Goetzke K, Hennig T, Ochs BG, Aigner T and Richter W: Premature induction of hypertrophy during in vitro chondrogenesis of human mesenchymal stem cells correlates with calcification and vascular invasion after ectopic transplantation in SCID mice. Arthritis Rheum 54: 3254-3266, 2006.

6. Cui JH, Park SR, Park K, Choi BH and Min BH: Preconditioning of mesenchymal stem cells with low-intensity ultrasound for cartilage formation in vivo. Tissue Eng 13: 351-360, 2007.

7. Liu K, Zhou GD, Liu W, Zhang WJ, Cui L, Liu X, Liu TY and Cao Y: The dependence of in vivo stable ectopic chondrogenesis by human mesenchymal stem cells on chondrogenic differentiation in vitro. Biomaterials 29: 2183-2192, 2008.

8. Liu X, Zhou GD, Lu XJ, Liu TY, Zhang WJ, Liu W and Cao YL: Potential of chondrogenesis of bone marrow stromal cells co-cultured with chondrocytes on biodegradable scaffold: in vivo experiment with pigs and mice. Zhonghua Yi Xue Za Zhi 87: 1929-1933, 2007.

9. Zhou Q, Li QH, Dai G and Shi GH: Efficient isolation of chondrocytes from rabbit articular cartilage with three-step enzymatic digestion and observation of their biological characteristics during cultivation in vitro. Zhonghua Wai Ke Za Zhi 43: 522-526, 2005.

10. Zhou G, Liu W, Cui L, Wang X, Liu T and Cao Y: Repair of porcine articular osteochondral defects in non-weightbearing areas with autologous bone marrow stromal cells. Tissue Eng 12: 3209-3221, 2006. 
11. Liu TY, Zhou GD, Wei X, Wu XL, Chen FG, Cui L, Liu W and Cao YL: Influence of transforming growth factor-betal inducing time on chondrogenesis of bone marrow stromal cells (BMSCs): in vitro experiment with porcine BMSCs. Zhonghua Yi Xue Za Zhi 87: 2218-2222, 2007.

12. Bjornsson S: Simultaneous preparation and quantitation of proteoglycans by precipitation with alcian blue. Anal Biochem 210: 282-291, 1993.

13. Friedenstein AJ, Gorskaja JF and Kulagina NN: Fibroblast precursors in normal and irradiated mouse hematopoietic organs. Exp Hematol 4: 267-274, 1976.

14. Zou L, Zou X, Chen L, Li H, Mygind T, Kassem M and Bunger C: Multilineage differentiation of porcine bone marrow stromal cells associated with specific gene expression pattern. J Orthop Res 26: 56-64, 2008.

15. Liu X, Sun H, Yan D, Zhang L, Lv X, Liu T, Zhang W, Liu W, Cao Y and Zhou G: In vivo ectopic chondrogenesis of BMSCs directed by mature chondrocytes. Biomaterials 31: 9406-9414, 2010.

16. Lyu SR, Wu WT, Hou CC and Hsieh WH: Study of cryopreservation of articular chondrocytes using the Taguchi method. Cryobiology 60: 165-176, 2010.

17. Surrao DC, Khan AA, McGregor AJ, Amsden BG and Waldman SD: Can microcarrier expanded chondrocytes synthesize cartilaginous tissue in vitro? Tissue Eng Part A 17: 1959-1967, 2011.

18. Spees JL, Gregory CA, Singh H, Tucker HA, Peister A, Lynch PJ, Hsu SC, Smith J and Prockop DJ: Internalized antigens must be removed to prepare hypoimmunogenic mesenchymal stem cells for cell and gene therapy. Mol Ther 9: 747-756, 2004.
19. Gregory CA, Reyes E, Whitney MJ and Spees JL: Enhanced engraftment of mesenchymal stem cells in a cutaneous wound model by culture in allogenic species-specific serum and administration in fibrin constructs. Stem Cells 24: 2232-2243, 2006.

20. Le Blanc K, Tammik C, Rosendahl K, Zetterberg E and Ringden O: HLA expression and immunologic properties of differentiated and undifferentiated mesenchymal stem cells. Exp Hematol 31: 890-896, 2003.

21. Le Blanc K, Tammik L, Sundberg B, Haynesworth SE and Ringden O: Mesenchymal stem cells inhibit and stimulate mixed lymphocyte cultures and mitogenic responses independently of the major histocompatibility complex. Scand J Immunol 57: 11-20, 2003.

22. Aggarwal S and Pittengerv MF: Human mesenchymal stem cells modulate allogeneic immune cell responses. Blood 105: 1815-1822, 2005.

23. Sotiropoulou PA, Perez SA, Salagianni M, Baxevanis CN and Papamichail M : Cell culture medium composition and translational adult bone marrow-derived stem cell research. Stem Cells 24: 1409-1410, 2006.

24. Tang B, Zhu B, Liang YY, Bi LK, Chen B, Hu ZC, Zhang K and Zhu JY: Early escharectomy and concurrent composite skin grafting over human acellular dermal matrix scaffold for covering deep facial burns. Plast Reconstr Surg 127: 1533-1538, 2011.

25. Jiong C, Jiake C, Chunmao H, Yingen P, Qiuhe W, Zhouxi F and Xiangsheng F: Clinical application and long-term follow-up study of porcine acellular dermal matrix combined with autoskin grafting. J Burn Care Res 31: 280-285, 2010. 